\title{
Design and Research of Intelligent Grain Depot
}

\author{
Chen Bo ${ }^{a}$, Xue Xiaoqing ${ }^{b}$, Liu Ting ${ }^{c}$ \\ Physics and Electronic Engineering, Xianyang Normal University, China \\ achen_bo_16@163.coml, b136465361@qq.com, cwhiteliuting@163.com
}

Keywords: Intelligent, Grain depot, Sensor.

\begin{abstract}
The intelligent grain storage management system designed in this paper can accurately and quickly analyze and calculate the storage capacity of each grain depot, and collect the temperature, humidity, and concentration of specific gases such as $\mathrm{O}_{2}, \mathrm{CO}_{2}$, and $\mathrm{PH}_{3}$ in real time. Through the analysis of the collected information, food condensation, mildew, pests and the like can be effectively avoided, providing technical assurance and scientific basis for the safe storage of grain. The corresponding auxiliary decision-making system, through the analysis of grain storage information across the country, can accurately predict the situation of food supply and demand and dynamically monitor the food market conditions.
\end{abstract}

\section{Introduction}

Grain is an important strategic commodity concerning national interest and people's livelihood. It has the bearing on social harmony and stability. Only by grasping the grain stock situation accurately can we correctly grasp the grain purchasing and storage, as well as the regulation and control, and consider the allocation and utilization of grain resources ${ }^{[1]}$. With the rise of "big data" and "cloud computing", the concept of "intelligent food" is constantly being mentioned. The so-called "intelligent food" mainly reflects the comprehensive data collection of grain depots, intelligent information processing, and information dissemination in real time. China's existing grain depot systems are mostly low as for intelligent level, data collection standards are not unified, statistical report is the main means while the data is single and difficult to ensure authenticity; in information processing, most of the relevant data collection, storage, processing and analysis of information are unified, information island phenomenon is serious; and in the information release, the data information is only open to superior departments rather than the inferiors, only to the government rather than the market, only to the local rather than overall China, and the information lag is rather serious. There is an urgent need for a set of intelligent monitoring and management systems to achieve real-time monitoring and integrated management of food stocks and food security ${ }^{[2]}$.

\section{Hardware overall design}

The system uses two levels of hierarchical structure: upper computer and lower computer. The upper computer is held by the computer, responsible for the management of the data collected by lower computer, mainly data display, query, data analysis, report printing, alarm and other functions. The lower computer consists of the main control module, the data acquisition module and the communication module ${ }^{[3]}$. The data acquisition module is responsible for collecting the food quality and the temperature, humidity, specific gas concentration such as $\mathrm{O}_{2}, \mathrm{CO}_{2}, \mathrm{PH}_{3}$ and others in the grain depot, sending the information collected by each sensor to the main control module. Then, through the wireless communication, the main control module sends the data to the upper computer for data analysis and decision-making. If the temperature in the grain depot is too high or the humidity is too big, the server will automatically open the grain depot air conditioner to lower the temperature or open the blower to reduce the humidity. The frame diagram of the system is shown in Figure 1. 


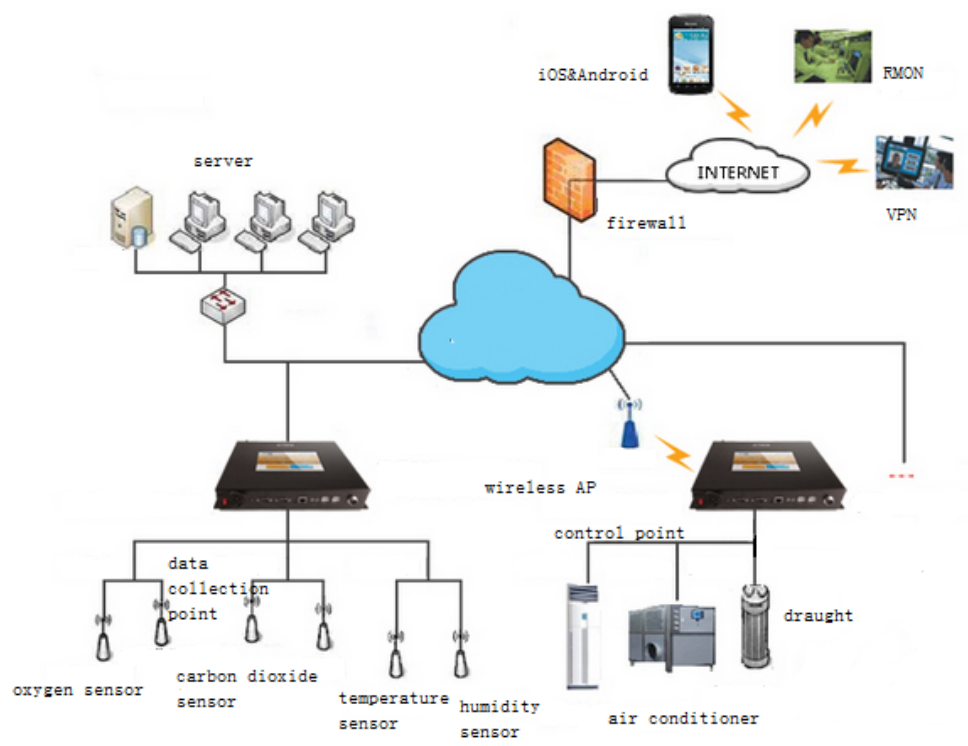

Figure.1 System construction diagrams

The main control module uses ATMEL's ATmega128. It belongs in ATMEL's 8-bit series of single-chip with the highest configuration and high stability and the application is extremely extensive. ATmega128 is used in the connection of the pressure sensor, temperature sensor, $\mathrm{O}_{2}$, $\mathrm{CO}_{2}, \mathrm{PH}_{3}$ and other gas concentration sensor; it receives the acquisition command from the host and sends the data back to the host upon implementation; its wireless communication protocol with the host is custom; it supports relay forwarding function and can be used for long-distance transmission.

Bridge sensor is used for grain weighing. It is very suitable for grain storage environment because of its large weighing range, anti-partial load, high precision, low power consumption and fully sealed characteristics. According to different types of granary, different numbers of bridge sensors can be used to establish corresponding weight models to meet the needs of various types of granary weighing.

Temperature sensor adopts the United States Dallas Company's DS18B20 digital temperature sensor; a cable can be mounted on multiple temperature sensors, taking less I / O port of the control chip. Its detection speed and accuracy is far more than analog temperature sensor.

Humidity sensor uses Switzerland SENSIRION Company's SHT15 moderate sensor, which has two-wire serial interface, strong anti-interference ability, fast response and digital signal output; the measuring range is $0-100 \% \mathrm{RH}$ and communicate with micro-controller through the I2C.

Sensors that detect gas concentrations are also corresponding mature products, such as O2-A2 sensor made in UK for detecting $\mathrm{O}_{2}$ concentration, as shown in Figure 2; MG811 sensor made in China for detecting $\mathrm{CO}_{2}$ concentration, as shown in Figure 3; BW SR-P04 PH3 sensor made in Canada for detecting $\mathrm{PH}_{3}$ concentration, as shown in Figure 4. These sensors are fully applicable to the grain storage environment for their high measurement accuracy and stability.

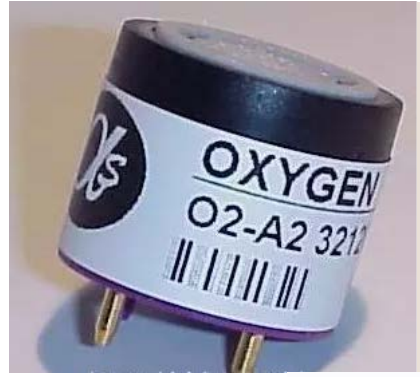

Figure.2 oxygen sensor
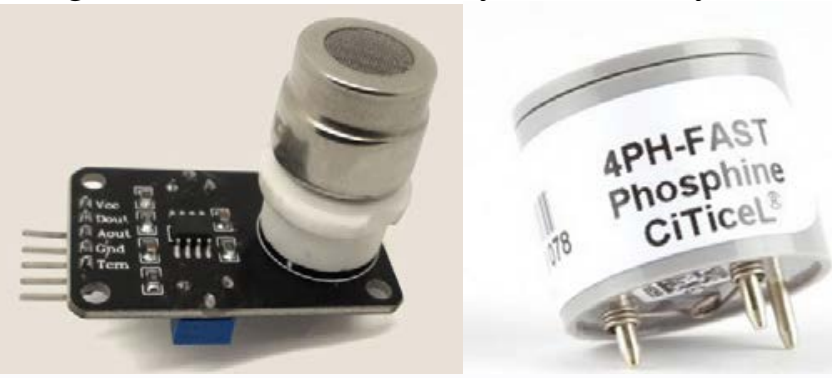

The wireless communication module mainly uses the UTC4432 wireless module produced by Hangzhou Feituo Electronic Technology Company to realize wireless communication. Responsible for all data collection and extension of the data aggregation, taking into account the large amount of data to be processed, and the late use of the touch screen requires functional expansion, ARM-based 
STM32 control chip is used.

\section{Design of Information Fusion Model for Granary Ventilation Decision}

Intelligent ventilation need to consider various grain situations, each kind of grain situation cannot alone as a condition to decide which ventilation mode to use, and ventilation conditions and calculation results may also support different ventilation modes. Therefore, in order to make the ventilation decision more correct and reasonable, it is required to make full use of all the grain information. Information fusion techniques can fuse multiple, homogeneous, and heterogeneous information, resulting in more valuable judgments than just a single message. In this paper, the use of intelligent ventilation system for computer-aided decision-making introduces air temperature, moisture, $\mathrm{O}_{2}, \mathrm{CO}_{2}, \mathrm{PH}_{3}$ and other sources of food information, to establish a fusion model, as shown in Figure 5.

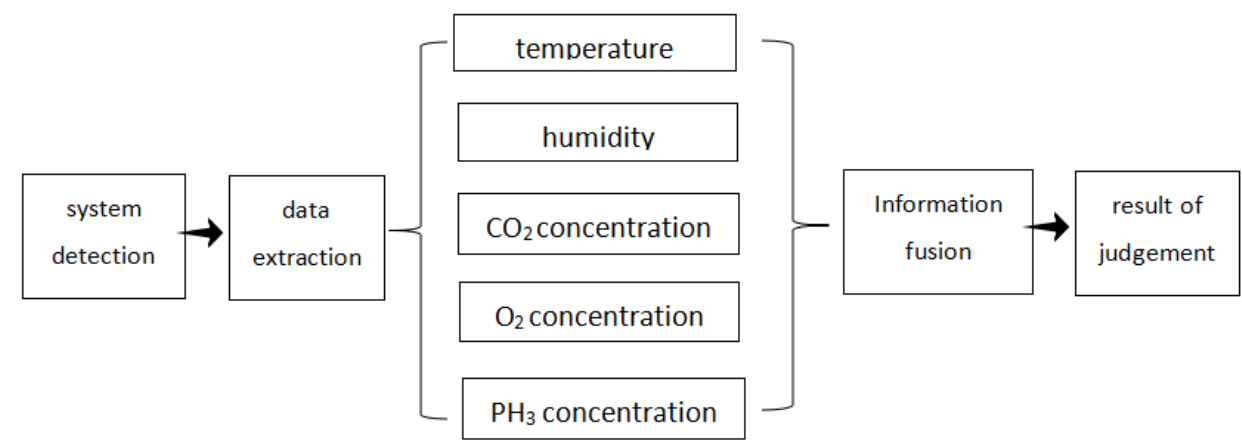

Figure 5 information fusion and judgement model

The fusion model can fuse the multiple grain status information obtained from the real-time grain situation and make the ventilation mode decision. Considering the influence of non-real data caused by sensor distortion drift, it is possible to collect the fusion of different sets of data at different time points and spatial points according to certain acquisition strategy on the basis of real-time data, in order to get more accurate fusion results. D-S evidence theory, as a fusion algorithm used for decision fusion, has been expanding since its introduction. D-S evidence theory emphasizes the reasoning of evidence, which can be regarded as a decision model itself and can be used to solve problems of decision-making. In this paper, based on the existing intelligent ventilation system, the decision-making method of ventilation mode is improved, D-S evidence theory is introduced into the decision-making of ventilation mode, and the ventilation mode is fused and judged.

\section{Conclusions}

Through the monitoring and analysis of temperature, humidity, $\mathrm{O}_{2}, \mathrm{CO}_{2}, \mathrm{PH}_{3}$ and other indicators, intelligent control of the granary fan, air conditioner, cold chiller is automatically realized. management costs is saved, management efficiency is improve, and food storage security is effectively guaranteed.

\section{Acknowledgments}

This work was financially supported by the Xianyang Science and Technology Research Project (2015k02-05) and the Special Project of Xianyang Normal University (13XSYK013).

\section{References}

[1] K- C Fan, A non - contact Automatic Measurement for Free - form Surface Profiles[J]. Computer Integrated Manufacturing System. 2016.3(4),277-285

[2] Gongchang Ren ,Yong Yang , Haichun Guo .Intelligent grain storage measurement system 
design and research[J]. 2011 International Conference on Frontiers of mechanical engineering and materials engineering, 2012:1881-1885

[3] Curotto,C.L etc. Multi-relational data mining in Microsoft SQL Server 2015[J]. WIT Transactions on Information and Communication Technologies, 2016, 12(2): 151-160. 